- Original Article

\title{
Relationship between Sedentary Time and Handgrip Strength in Healthy Korean Women: Korea National Health and Nutrition Examination Survey 2014-2016
}

\author{
Seung-Yeon Lee, Da-Hye Son, Yong-Jae Lee* \\ Department of Family Medicine, Yonsei University College of Medicine, Seoul, Korea
}

Background: This study aimed to examine the association between sitting time and handgrip strength in healthy Korean women.

Methods: A total of 5,437 participants were included from the Korea National Health and Nutrition Examination Survey 2014-2016. The overall daily sitting time was estimated using health interview surveys, and handgrip strength was assessed using a digital hand dynamometer. The relationship between sitting time and handgrip strength was calculated with a weighted analysis of covariance after adjusting for confounding variables.

Results: Participants in each age group (19-39, 40-64, $\geq 65$ years) were divided into three categories according to sitting time: $\leq 5,6-9$, and $\geq 10 \mathrm{~h} / \mathrm{d}$. The handgrip strength tended to decrease as sitting time increased after adjusting for age, body mass index, alcohol intake, cigarette smoking, resistance exercise, aerobic physical activity, household income, education level, hypertension, diabetes mellitus, dyslipidemia, and depression in all age groups (all $\mathrm{P}<0.001)$.

Conclusion: We observed the inverse relationship between sitting time and handgrip strength in healthy Korean women.

Keywords: Sedentary Behavior; Hand Strength; Surveys and Questionnaires; Physical Examination

Received: January 26, 2019, Revised: July 23, 2019, Accepted: July 23, 2019

*Corresponding Author: Yong-Jae Lee https://orcid.org/0000-0002-6697-476X

Tel: +82-2-2019-2630, Fax: +82-2-3462-8209, E-mail: ukyjhome@yuhs.ac 


\section{INTRODUCTION}

The World Health Organization (WHO) has announced that physical inactivity can have grave implications for public health and considers sedentary lifestyle to be among the ten leading causes of mortality and disability worldwide in 2002. The term "sedentary," Latin word for "sedere," means "to sit"; thus, the term "sedentary lifestyle" is used to describe behaviors involving sedentary and low levels of energy expenditure, not only leisure sedentary time, including watching movies, but also productive sedentary time, including paperwork in an office. ${ }^{1,2)}$ In recent years, sedentary behavior has increased dramatically with a change in modern lifestyles. The number of manual workers has substantially reduced owing to technological developments, mechanization, and automation. Moreover, household chores have been simplified by household electrical appliances, which make things easier and reduce physical activity. ${ }^{2)}$ Physical inactivity increases the risk of several detrimental health issues, either directly or indirectly. Furthermore, previous studies have shown that increased sedentary time carries health risks of its own, independent of physical activity. ${ }^{1,3,4)}$ A dose-response association between sedentary time and mortality due to all causes and cardiovascular disease (CVD) was shown in a prospective study in Canada. ${ }^{3)}$ Another study showed that sedentary lifestyle in the elderly was associated with decreased muscle mass and increased risk of sarcopenia, independent of physical activity.

Sarcopenia is defined as the loss of muscle function and muscle mass. Sarcopenia is now regarded a major health issue, and the associated public health burden leads to obesity, osteoporosis, metabolic health, decreased quality of life, physical disabilities, depression and, ultimately, death. ${ }^{5)}$ To diagnose sarcopenia, muscle function, such as muscle strength and physical performance, and muscle mass should be assessed together. Measurement of handgrip strength is considered an easy and simple way to measure muscle function; thus, it is used to diagnose sarcopenia worldwide.

Previous studies on Korean people have reported on the association between sedentary time and development of health problems. However, these studies focused mainly on chronic diseases, such as CVD and type 2 diabetes. This study aimed to evaluate the association between sedentary time and sarcopenia measured by handgrip strength in healthy Korean women.

\section{METHODS}

\section{Survey Overview and Study Population}

This cross-sectional study used data acquired from the 2014-2016 Korea National Health and Nutrition Examination Survey (KNHANES). This consists of a nutrition survey, a health interview survey, and a health examination survey and was devised in 1998 to estimate the health and nutritional status of the Korean population. The Korea Centers for Disease Control and Prevention (KCDC) conducted this nationally representative survey. A total of 5,437 female participants, older than 19 years, were included from the 2014-2016 KNHANES. We excluded women whose sedentary time and/or handgrip strength data were missing; who were pregnant; who had a history of cancer or chronic diseases, which could influence sedentary time, such as hepatitis B, hepatitis C, liver cirrhosis, chronic kidney disease, angina, myocardial infarction, asthma, thyroid disease, osteoarthritis and rheumatoid arthritis; or who had a limitation of physical activity due to any reason. Participants were divided in to three groups according to age.

Table 1. Demographic and clinical characteristics according to age (years)

\begin{tabular}{|c|c|c|c|c|}
\hline \multirow{2}{*}{ Characteristic } & \multicolumn{3}{|c|}{ Age group (y, N=5,437) } & \multirow{2}{*}{ P-value } \\
\hline & $19-39$ & $40-64$ & $\geq 65$ & \\
\hline No. of patients & 1,978 & 2,664 & 795 & \\
\hline Age (y) & $29.7 \pm 0.2$ & $50.1 \pm 0.2$ & $73.1 \pm 0.2$ & 0.11 \\
\hline Sedentary time $(\mathrm{h} / \mathrm{d})$ & $26.2 \pm 0.1$ & $26.1 \pm 0.1$ & $20.5 \pm 0.2$ & 0.10 \\
\hline Body mass index $\left(\mathrm{kg} / \mathrm{m}^{2}\right)$ & $22.1 \pm 0.1$ & $23.4 \pm 0.1$ & $24.0 \pm 0.1$ & 0.07 \\
\hline Cigarette smoking (\%) & $13.5 \pm 1.0$ & $6.9 \pm 0.6$ & $6.3 \pm 1.0$ & $<0.001$ \\
\hline Alcohol ingestion (\%) & $15.2 \pm 0.9$ & $12.5 \pm 0.8$ & $5.3 \pm 0.9$ & $<0.001$ \\
\hline Aerobic physical activity (\%) & $55.0 \pm 1.4$ & $50.3 \pm 1.1$ & $28.7 \pm 2.0$ & $<0.001$ \\
\hline Resistance exercise (\%) & $9.5 \pm 0.8$ & $9.8 \pm 0.6$ & $6.3 \pm 1.1$ & 0.06 \\
\hline Household income quartile (\%) & & & & $<0.001$ \\
\hline 1 (Lowest) & $0.9 \pm 0.2$ & $10.7 \pm 0.7$ & $73.0 \pm 2.1$ & \\
\hline 2 & $1.4 \pm 0.3$ & $12.1 \pm 0.7$ & $11.2 \pm 1.4$ & \\
\hline 3 & $35.6 \pm 1.3$ & $46.2 \pm 1.3$ & $10.0 \pm 1.3$ & \\
\hline 4 (Highest) & $62.1 \pm 1.4$ & $30.9 \pm 1.4$ & $5.8 \pm 1.1$ & \\
\hline Education (\%) & & & & $<0.001$ \\
\hline$\leq$ Elementary school & $0.9 \pm 0.2$ & $10.7 \pm 0.7$ & $73.0 \pm 2.1$ & \\
\hline Middle school & $1.4 \pm 0.3$ & $12.1 \pm 0.7$ & $11.2 \pm 1.4$ & \\
\hline High school & $35.6 \pm 1.3$ & $46.2 \pm 1.3$ & $10.0 \pm 1.3$ & \\
\hline$\geq$ University & $62.1 \pm 1.4$ & $30.9 \pm 1.4$ & $5.8 \pm 1.1$ & \\
\hline
\end{tabular}

Values are presented as number or mean \pm standard deviation. 


\section{Data Collection of Study Population}

During the 2014-2016 KNHANES, the participants were notified that their household has been randomly selected to participate voluntarily in a nationwide representative survey performed by the KCDC, and written informed consent was acquired from all participants. All citizens who agreed to participate had the right to withdraw at any time as per the National Statistics Law of Korea. The 2014-2016 KNHANES collected demographic, social, and health data of these participants. Covariates such as age, educational level, and household income were considered as socioeconomic factors. Health-related factors included alcohol intake, cigarette smoking, resistance exercise, and aerobic physical activity. Socioeconomic information was obtained from a health interview, while health-related behaviors were collected through a self-reported questionnaire. Current smoker was defined as current smoking status or those who had smoked more than 100 cigarettes during their lifetime. Alcohol intake was defined as more than 2-3 drinks per week based on queries on the participants' average alcohol consumption frequency. Aerobic physical activity was measured according to the performance of aerobic activity as recommended by the WHO guidelines. Those who participated in at least 150 minutes or more of moderate-intensity activity per week or a combination of moderate- and high-intensity activity for 75 minutes or more per week were classified as "meets recommended guidelines"; all others were classified as "under recommended". With regard to resistance exercise, participants were asked "How many days per week do you perform resistance exercises, such as push-ups, sit-ups, dumbbell exercises, weight lifting, and horizontal bar lifting?" The resistance exercise group consisted of subjects who performed resistance exercise 3 times or more per week. Trained medical staff performed the anthropometric measurements following a standardized procedure. Height and body weight were measured to the nearest $0.1 \mathrm{~cm}$ and $0.1 \mathrm{~kg}$ while participants wore light clothing without shoes. BMI was calculated as weight $(\mathrm{kg})$ divided by the square of height $\left(\mathrm{m}^{2}\right)$. Handgrip strength was measured using a digital hand dynamometer (Digital Grip Dynamometer, TKK 5401; Takei Scientific Instruments Co. Ltd, Tokyo, Japan) and was measured with the subject in a standing position with their arms fully extended at the sides, not touching the body. The participants were asked to exert maximum grip strength for $<3$ seconds, 3 times each with their left and right hands. At least 30 seconds of rest interval was allowed between each trial. Handgrip strength was defined as the maximally measured grip strength of the dominant hand. ${ }^{6}$ ) The overall daily sedentary time was estimated using the health interview survey, particularly the following question: "on average, how many hours per day do you sit down?" Responses were divided into three categories according to sedentary time: Q1, $\leq 5 \mathrm{~h} / \mathrm{d} ; \mathrm{Q} 2,6-9 \mathrm{~h} / \mathrm{d}$; and $\mathrm{Q} 3, \geq 10 \mathrm{~h} / \mathrm{d}$ considering the criteria of a previous study and population distribution in each age group. ${ }^{7)}$

\section{Statistical Analysis}

Sampling weights were used to account for the complex survey design of the KNHANES; therefore, we obtained valid estimates that represented all Korean female adults and avoided biased estimates. The characteristics of the study participants were analyzed according to age and sedentary time using a weighted one-way analysis of variance for continuous variables and a weighted chi-square test for categorical variables.

The trend and relationship of handgrip strength and sedentary time (h/d) were calculated with a weighted analysis of covariance. All statistical analyses were performed using IBM SPSS statistical software ver. 23.0 (IBM Corp., Armonk, NY, USA). A P-value $<0.05$ was considered significant.

Table 2. Demographic and clinical characteristics according to sedentary time (h/d)

\begin{tabular}{|c|c|c|c|c|}
\hline \multirow{2}{*}{ Characteristic } & \multicolumn{3}{|c|}{ Sedentary time ( $h / d, N=5,437$ ) } & \multirow{2}{*}{ P-valu } \\
\hline & $\leq 5$ & $6-9$ & $\geq 10$ & \\
\hline No. of patients & 1,655 & 1,607 & 2,175 & \\
\hline Age $(y)$ & $45.8 \pm 0.4$ & $43.4 \pm 0.4$ & $42.3 \pm 0.4$ & $<0.001$ \\
\hline Body mass index $\left(\mathrm{kg} / \mathrm{m}^{2}\right)$ & $23.1 \pm 0.1$ & $22.9 \pm 0.1$ & $22.9 \pm 0.1$ & $<0.001$ \\
\hline Cigarette smoking (\%) & $26.8 \pm 2.3$ & $25.3 \pm 2.2$ & $48.0 \pm 2.6$ & 0.02 \\
\hline Alcohol ingestion (\%) & $13.2 \pm 0.9$ & $13.4 \pm 1.0$ & $12.5 \pm 0.8$ & 0.7 \\
\hline Aerobic physical activity (\%) & $55.5 \pm 1.6$ & $49.2 \pm 1.4$ & $47.2 \pm 1.4$ & $<0.001$ \\
\hline Resistance exercise (\%) & $10.2 \pm 0.9$ & $9.8 \pm 0.9$ & $8.3 \pm 0.7$ & 0.17 \\
\hline Household income quartile (\%) & & & & $<0.001$ \\
\hline 1 (Lowest) & $11.1 \pm 0.9$ & $11.7 \pm 1.0$ & $12.9 \pm 0.9$ & \\
\hline 2 & $28.7 \pm 1.4$ & $23.2 \pm 1.3$ & $21.3 \pm 1.2$ & \\
\hline 3 & $31.7 \pm 1.4$ & $31.6 \pm 1.6$ & $29.5 \pm 1.3$ & \\
\hline 4 (Highest) & $28.5 \pm 1.5$ & $33.5 \pm 1.6$ & $36.3 \pm 1.5$ & \\
\hline Education (\%) & & & & $<0.001$ \\
\hline$\leq$ Elementary school & $14.3 \pm 1.0$ & $12.1 \pm 0.9$ & $9.8 \pm 0.8$ & \\
\hline Middle school & $10.2 \pm 0.8$ & $7.6 \pm 0.7$ & $4.4 \pm 0.5$ & \\
\hline High school & $40.4 \pm 1.5$ & $36.2 \pm 1.5$ & $38.2 \pm 1.3$ & \\
\hline$\geq$ University & $35.1 \pm 1.4$ & $44.1 \pm 1.6$ & $47.6 \pm 1.5$ & \\
\hline
\end{tabular}

Values are presented as number or mean \pm standard deviation. 


\section{RESULTS}

Table 1 shows the participants' general characteristics according to age. The total number of participants was 5,437. The proportion of cigarette smoking was highest in the 40-64-year-age group. The proportion of alcohol intake and aerobic physical activity was highest in the 19-39-year-age group. Participants in the 19-39-year-age group had the highest socioeconomic position, according to their household income and education level. Table 2 shows the demographic and clinical characteristics of 5,437 women according to sedentary time. The mean age, BMI, and proportion of aerobic physical activity decreased as sedentary time increased in women. The group who sat down for more than $10 \mathrm{~h} / \mathrm{d}$ on average had the highest socioeconomic position, according to their household income and education level. Table 3 presents the independent relationship between handgrip strength and sedentary time according to the age group of participants: 19-39, 4064 years, and $\geq 65$ years, and the oldest participant was 80 years old. Handgrip strength tended to decrease as sedentary time increased after adjusting for age, BMI, alcohol ingestion, cigarette smoking, resistance exercise, aerobic physical activity, education level, household income, hypertension, diabetes mellitus, dyslipidemia, and depression.

\section{DISCUSSION}

In this large, representative sample of healthy Korean women, we found that sedentary time was inversely associated with handgrip strength. Our findings are in agreement with the results of previous studies. Many studies have shown that physical inactivity is generally associated with sarcopenia, ${ }^{8-10)}$ and resistance exercise is considered the best to prevent sarcopenia. ${ }^{11,12)}$ Physical activity, such as walking, has been shown to be associated with a decreased risk of sarcopenia, as assessed by a dual energy X-ray absorptiometry in Korean elderly men from the KNHANES IV 2, 3 (2008-2009). ${ }^{13)}$ Measurement of handgrip strength is a fast, simple, standard, and reliable scale to measure overall voluntary muscle strength, and it is considered an important value to define sarcopenia, as weak handgrip strength is associated with a low muscle mass and predictive of poor physical performance. ${ }^{14,15)}$

In this nationally representative cross-sectional study, we found that handgrip strength is inversely associated with sedentary time in the healthy Korean women regardless of age. This inverse association was maintained after controlling for potential confounding variables including age, BMI, cigarette smoking, alcohol ingestion, aerobic physical activity, resistance exercise, household income, education level, hypertension, diabetes mellitus, dyslipidemia, and depression. If sedentary time is increased, physical activity is decreased; the risk of sarcopenia is also increased, and it could be delineated by a weak handgrip strength. ${ }^{16)}$

This study has certain limitations that should be considered when interpreting the findings. Firstly, it is difficult to establish a causal relationship between sedentary time and handgrip strength in healthy Korean women as this study used a cross-sectional design. Thus, prospectively designed studies are necessary to clarify the relationship between sedentary time, sarcopenia, and handgrip strength. Secondly, this study was based on a questionnaire survey; thus, we could not fully exclude the effect of information bias. Self-reported sedentary time may be less accurate than objectively measured sedentary time.

Table 3. Trend analysis of handgrips strength $(\mathrm{kg})$ and sedentary time $(\mathrm{h} / \mathrm{d})(\mathrm{N}=5,437)$

\begin{tabular}{|c|c|c|c|c|c|}
\hline \multirow{2}{*}{ Age (y) } & \multirow{2}{*}{ Category } & \multicolumn{3}{|c|}{ Sedentary time $(\mathrm{h} / \mathrm{d})$} & \multirow{2}{*}{ P-value } \\
\hline & & $\leq 5$ & $6-9$ & $\geq 10$ & \\
\hline \multirow[t]{5}{*}{$19-39(N=1,978)$} & No. of patients & 481 & 603 & 894 & \\
\hline & Unadjusted & $26.6 \pm 0.2$ & $26.3 \pm 0.2$ & $25.9 \pm 0.2$ & $<0.001$ \\
\hline & Model $1^{*}$ & $26.4 \pm 0.2$ & $26.3 \pm 0.2$ & $26.0 \pm 0.2$ & $<0.001$ \\
\hline & Model $2^{\dagger}$ & $27.2 \pm 0.5$ & $27.1 \pm 0.5$ & $26.8 \pm 0.4$ & $<0.001$ \\
\hline & Model $3^{\ddagger}$ & $27.0 \pm 1.3$ & $27.0 \pm 1.3$ & $26.6 \pm 1.3$ & $<0.001$ \\
\hline \multirow[t]{5}{*}{$40-64(N=2,664)$} & No. of patients & 970 & 767 & 927 & \\
\hline & Unadjusted & $26.3 \pm 0.2$ & $26.1 \pm 0.2$ & $25.9 \pm 0.2$ & $<0.001$ \\
\hline & Model $1^{\star}$ & $26.4 \pm 0.2$ & $26.2 \pm 0.2$ & $25.9 \pm 0.2$ & $<0.001$ \\
\hline & Model $2^{\dagger}$ & $26.9 \pm 0.3$ & $26.7 \pm 0.3$ & $26.5 \pm 0.3$ & $<0.001$ \\
\hline & Model $3^{\ddagger}$ & $27.0 \pm 1.3$ & $27.0 \pm 1.3$ & $26.6 \pm 1.3$ & $<0.001$ \\
\hline \multirow[t]{5}{*}{$\geq 65(\mathrm{~N}=795)$} & No. of patients & 204 & 237 & 354 & \\
\hline & Unadjusted & $21.7 \pm 0.4$ & $21.2 \pm 0.4$ & $19.3 \pm 0.3$ & $<0.001$ \\
\hline & Model $1^{*}$ & $20.8 \pm 0.3$ & $20.5 \pm 0.3$ & $20.2 \pm 0.3$ & $<0.001$ \\
\hline & Model $2^{\dagger}$ & $22.5 \pm 0.7$ & $22.2 \pm 0.8$ & $22.0 \pm 0.7$ & $<0.001$ \\
\hline & Model $3^{\ddagger}$ & $22.5 \pm 0.7$ & $22.2 \pm 0.8$ & $22.0 \pm 0.7$ & $<0.001$ \\
\hline
\end{tabular}

Values are presented as number or mean \pm standard deviation assessed by analysis of variance test.

BMI, body mass index.

"Model 1: adjusted for age and BMI. 'Model 2: adjusted for age, BMl, cigarette smoking, alcohol ingestion, aerobic physical activity, resistance exercise, household income, and education. ₹Model 3: adjusted for age, BMl, cigarette smoking, alcohol ingestion, aerobic physical activity, resistance exercise, household income, education level, hypertension, diabetes mellitus, dyslipidemia, and depression. 
Thirdly, the questions used here did not distinguish between sedentary leisure time, such as time spent playing video games or watching television, and productive sedentary time, such as working on the computer. Since physical activity can be differentially associated with different types of sedentary pursuits, ${ }^{17)}$ measurement of the total sedentary time without distinguishing between sedentary leisure time and productive sedentary time could affect the results. Finally, as there were no data on muscle mass in the 2014-2016 KNHANES, we had to use only handgrip strength and consider it to be a representative value of sarcopenia. However, even though handgrip strength has been widely accepted as the most efficient value for defining sarcopenia in recent years, several guidelines from European Groups Working on Sarcopenia in Older People (the Sarcopenia Working Group) or Asian Working Group for Sarcopenia still recommend the use of both muscle mass and muscle strength to diagnose sarcopenia. Therefore, further studies on the relationship between sedentary time and muscle mass in healthy Korean adults will be helpful to clarify the present study results.

Despite these potential limitations, to the best of our knowledge, this is the first population-based study to demonstrate a relationship between sedentary time and handgrip strength in healthy women. In conclusion, we demonstrated a significant inverse association between sedentary time and handgrip strength that may be associated with sarcopenia. Thus, public health intervention to reduce sedentary time or improve national physical performance is required to form a preventive perspective of sarcopenia.

\section{CONFLICT OF INTEREST}

No potential conflict of interest relevant to this article was reported.

\section{ORCID}

Seung-Yeon Lee: https://orcid.org/0000-0002-6904-6635

Da-Hye Son: https://orcid.org/0000-0001-7464-996X

Yong-Jae Lee: https://orcid.org/0000-0002-6697-476X

\section{REFERENCES}

1. Owen N, Healy GN, Matthews CE, Dunstan DW. Too much sitting: the population health science of sedentary behavior. Exerc Sport Sci Rev 2010;38:105-13.

2. Inyang MP, Stella OO. Sedentary lifestyle: health implications. IOSR J Nurs Health Sci 2015;4:20-5.

3. Katzmarzyk PT, Church TS, Craig CL, Bouchard C. Sitting time and mortality from all causes, cardiovascular disease, and cancer. Med Sci
Sports Exerc 2009;41:998-1005.

4. Gianoudis J, Bailey CA, Daly RM. Associations between sedentary behaviour and body composition, muscle function and sarcopenia in community-dwelling older adults. Osteoporos Int 2015;26:571-9.

5. Beaudart C, Zaaria M, Pasleau F, Reginster JY, Bruyere O. Health outcomes of sarcopenia: a systematic review and meta-analysis. PLoS One 2017;12:e0169548.

6. Yoo JI, Choi H, Ha YC. Mean hand grip strength and cut-off value for sarcopenia in Korean adults using KNHANES VI. J Korean Med Sci 2017;32:868-72.

7. An SY, Kang HE, Kim SM, Sok SA, Yang HJ, Lee GN. Association between sitting time and diabetes mellitus, cardiovascular disease and metabolic syndrome in Korean: the sixth Korean National Health and Nutrition Examination Survey 2013 and 2014. Korean J Fam Pract 2017;7:588-95.

8. Evans WJ. Skeletal muscle loss: cachexia, sarcopenia, and inactivity. Am J Clin Nutr 2010;91:1123S-1127S.

9. Park H, Park S, Shephard RJ, Aoyagi Y. Yearlong physical activity and sarcopenia in older adults: the Nakanojo Study. Eur J Appl Physiol 2010;109:953-61.

10. Akune T, Muraki S, Oka H, Tanaka S, Kawaguchi H, Nakamura K, et al. Exercise habits during middle age are associated with lower prevalence of sarcopenia: the ROAD study. Osteoporos Int 2014;25:1081-8.

11. Steffl M, Bohannon RW, Sontakova L, Tufano JJ, Shiells K, Holmerova I. Relationship between sarcopenia and physical activity in older people: a systematic review and meta-analysis. Clin Interv Aging 2017;12:835-45.

12. Aagaard P, Suetta C, Caserotti P, Magnusson SP, Kjaer M. Role of the nervous system in sarcopenia and muscle atrophy with aging: strength training as a countermeasure. Scand J Med Sci Sports 2010;20:49-64.

13. Kim SH, Kim TH, Hwang HJ. The relationship of physical activity (PA) and walking with sarcopenia in Korean males aged 60 years and older using the fourth Korean National Health and Nutrition Examination Survey (KNHANES IV-2, 3), 2008-2009. Arch Gerontol Geriatr 2013;56:472-7.

14. Bohannon RW. Muscle strength: clinical and prognostic value of hand-grip dynamometry. Curr Opin Clin Nutr Metab Care 2015;18:465-70.

15. Granic A, Jagger C, Davies K, Adamson A, Kirkwood T, Hill TR, et al. Effect of dietary patterns on muscle strength and physical performance in the very old: findings from the Newcastle 85+ study. PLoS One 2016;11:e0149699.

16. Martinez-Gonzalez MA, Martinez JA, Hu FB, Gibney MJ, Kearney J. Physical inactivity, sedentary lifestyle and obesity in the European Union. Int J Obes Relat Metab Disord 1999;23:1192-201.

17. Feldman DE, Barnett T, Shrier I, Rossignol M, Abenhaim L. Is physical activity differentially associated with different types of sedentary pursuits? Arch Pediatr Adolesc Med 2003;157:797-802. 